\title{
The Investigation of Fluorescence Spectra and Fluorescence Quantum Yield of Enrofloxacin
}

\author{
Lihua Ma, Baosheng Liu ", Chundan Wang, Hongcai Zhang, Xu Cheng \\ College of Chemistry and Environmental Science, Hebei University, Baoding, China
}

Email address:

lbs@hbu.edu.cn (Baosheng Liu)

${ }^{*}$ Corresponding author

\section{To cite this article:}

Lihua Ma, Baosheng Liu, Chundan Wang, Hongcai Zhang, Xu Cheng. The Investigation of Fluorescence Spectra and Fluorescence Quantum Yield of Enrofloxacin. Journal of Chemical, Environmental and Biological Engineering. Vol. 2, No. 1, 2018, pp. 11-16. doi: 10.11648/j.jcebe.20180201.13

Received: May 4, 2018; Accepted: May 30, 2018; Published: June 1, 2018

\begin{abstract}
In this paper, the fluorescence spectra of Enrofloxacin (ENR) in different pH conditions was studied in order to determine its structural changes due to protonation with $\mathrm{pH}$ changes. The ENR two-step dissociation constant is calculated and the fluorescence quantum yield under acidic conditions is measured. In the strong acidic conditions, ENR exists of $\mathrm{H}_{3} \mathrm{~L}^{2+}$ form of which maximum emission wavelength is at $450 \mathrm{~nm}$. At the condition of pH 2.45 to 4.23 , ENR exists of $\mathrm{H}_{2} \mathrm{~L}^{+}$form with strong and steady fluorescence. The maximum emission wavelength is still $450 \mathrm{~nm}$. At the condition of $\mathrm{pH}$ more than 4.23 , the maximum emission wavelengths are gradually blue shifted to $445 \mathrm{~nm}$ and the fluorescence intensity decrease with the increase of $\mathrm{pH}$ which shows that $\mathrm{H}_{2} \mathrm{~L}^{+}$loses one proton with the increase of $\mathrm{pH}$ and exists in the form of bipolar ion HL. When the $\mathrm{pH}$ is more than 12.28 , the fluorescence intensity are weakened to nearly disappear with the increase of $\mathrm{pH}$ value, indicating that $\mathrm{HL}$ gradually loses the proton with the conversion to the anion of $\mathrm{L}^{-}$which is weaker fluorescence. In the buffer solution of $\mathrm{pH} 3.00$, with quinine sulfate as reference, the fluorescence quantum yield of ENR at excitation wavelength of $274 \mathrm{~nm}$ is 0.125 .
\end{abstract}

Keywords: Enrofloxacin, Fluorescence Spectroscopy, Fluorescence Quantum Yield, Dissociation Constant

\section{Introduction}

Enrofloxacin (ENR) is a broad spectrum of fluoroquinolone (FQ) antibiotic with high physiological activity and among the most consumed quinolones on livestock in China $[1,2]$. The widely used quinolones will then accumulate in the food chain, causing environment pollution and would possibly lead to undesired effects in humans [3, 4]. As quinolones can be synthesized by chemical methods, modifying the structure of these drugs can significantly improve its shortcomings. Thus, quantitative and qualitative study of quinolones has a great significance.

ENR molecules contain a carboxyl group and a piperazinyl amine group and could exist as cationic, zwitterionic, and anionic species in solution depending on $\mathrm{pH}$ values [5], which emission is closely related to the degree of protonation $[6,7]$. Compared with NMR or IR, fluorescence spectroscopy has the advantages of fast speed and low cost of instruments. In this study, the fluorescence spectra of ENR under different $\mathrm{pH}$ conditions were investigated to determine the structural changes. The dissociation constants were calculated and the fluorescence quantum yields were measured by using quinine sulfate as reference solution. Chemical structure of ENR is shown as Figure 1.<smiles>CCN1CCN(c2cc3c(cc2F)c(=O)c(C(=O)O)cn3C2CC2)CC1</smiles>

Figure 1. Chemical structure of Enrofloxacin.

\section{Experimental}

\subsection{Apparatus and Materials}

All fluorescence spectra were recorded using a RF-5301PC spectrofluorophotometer (Shimadzu, Japan). Absorption was measured using a UV-visible spectrophotometer (UV-265, Shimadzu, Japan). All pH measurements were carried out with a pHS-3C precision acidity meter (Leici, Shanghai, China).

ENR (biochemical reagent, Shanghai Reagent Second 
Factory, China) solution (10 $\mu \mathrm{M})$ was prepared. A series of buffer solution was configured from pure borax, potassium dihydrogen phosphate, hydrochloric acid and sodium hydroxide (biochemical reagent, Shanghai Reagent Second Factory, China). Quinine sulfate (biochemical reagent, Shanghai Reagent Second Factory, China) solution was used at a concentration of $40 \mu \mathrm{M}$ with $0.05 \mathrm{M}$ sulfuric acid solution. The reagents used in the experiments were analytical grade, and the water was double-distilled water.

The investigation was approved and that informed consent was obtained.

\subsection{Procedures}

ENR solution $(1.0 \mathrm{~mL} ; 10 \mu \mathrm{M})$ was added to $10 \mathrm{~mL}$ colorimetric tubes. The samples were diluted to a scaled volume with $\mathrm{pH}$ buffer solution and mixed thoroughly by shaking. The excitation and emission slits were set at $5 \mathrm{~nm}$ and the excitation wavelength for ENR solution was $274 \mathrm{~nm}$. The fluorescence emission spectra of ENR were scanned.

\section{Results and Discussion}

\subsection{Fluorescence Spectra of ENR under Near-Neutral or Weakly Acidic Conditions}

The fluorescence spectra of ENR under the near-neutral or weakly acid conditions are shown in Figure 2. From In the figure, the emission peak was blue shifted and decreased significantly with increasing $\mathrm{pH}$. When $\mathrm{pH}$ was $4.23-4.94$, the maximum emission wavelength was $450 \mathrm{~nm}$. When $\mathrm{pH}$ was 5.84-7.78, the maximum emission wavelengths were blue shifted to $445 \mathrm{~nm}$. This spectral characteristic indicated that the presence of ENR varies with different $\mathrm{pH}$, and the fluorescence properties of these two forms are different, as shown in Figure 2.

The red shift or blue shift of the spectrum was related to the degree of conjugation of the molecule [8-10]. The phenomenon that the maximum emission wavelength was gradually blue shifted with increase in $\mathrm{pH}$, indicated that ENR should have a high degree of conjugation and a more rigid structure at lower $\mathrm{pH}$ values. Therefore, the proton dissociation equilibrium of ENR at this $\mathrm{pH}$ range is shown in Figure 3.

When $\mathrm{pH}$ was increased to 7.78 from 4.23, ENR formed a bipolar ion $\mathrm{HL}$ due to the presence of $\mathrm{H}_{2} \mathrm{~L}^{+}$that loses a proton from the carboxyl group bound oxygen. Because the proton of the carboxyl group bound oxygen can be combined with the 4-carbonyl oxygen in the quinoline ring through hydrogen, the substance can form a ring structure, and then conjugated with the quinoline ring. After lossing the proton, the conjugation of the whole molecule was reduced, causing a blue shift in fluorescence emission wavelength.

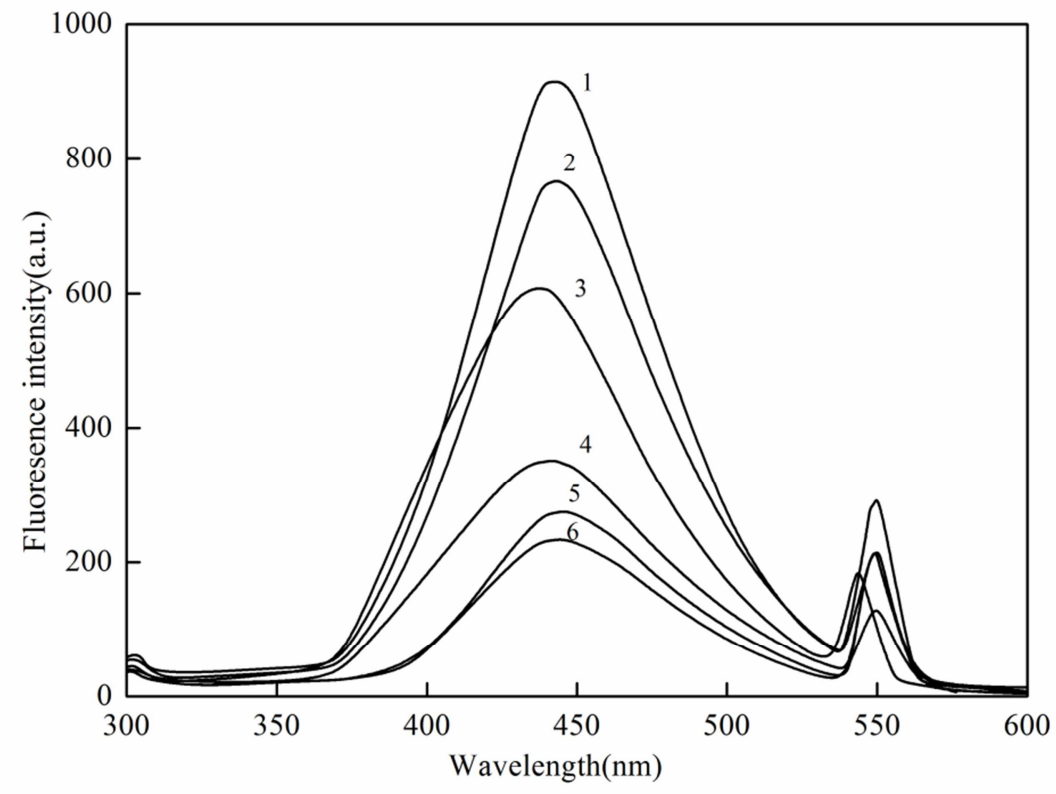

(1) $\mathrm{pH}=4.23$ (2) $\mathrm{pH}=4.94$ (3) $\mathrm{pH}=5.84$ (4) $\mathrm{pH}=6.67$ (5) $\mathrm{pH}=7.14$ (6) $\mathrm{pH}=7.78$

Figure 2. Fluorescence spectra of ENR under near-neutral or weakly acidic conditions.<smiles></smiles>

Figure 3. The process of $\mathrm{H}_{2} \mathrm{~L}^{+}$dissociation to $H L$. 


\subsection{Fluorescence Spectra of ENR in the Acidic Conditions}

The fluorescence spectra of ENR under acidic conditions are shown in Figure 4. The emission peaks at $450 \mathrm{~nm}$ was increased gradually with increasing $\mathrm{pH}$, and the position of maximum emission peak was basically unchanged. This result indicates that ENR may further combine with a proton under increasing acidic conditions, namely $\mathrm{H}_{2} \mathrm{~L}^{+}$to $\mathrm{H}_{3} \mathrm{~L}^{2+}$. The binding site of the proton on ENR may be at the 1-position nitrogen in the quinoline ring, or at the carbonyl oxygen. As
ENR fluorescence intensity was significantly increased under the condition as shown in Figure 4, the proton may be combined with the 1-position nitrogen in the quinoline ring within the $\mathrm{pH}$ range $1.02-2.81$, the proton dissociation equilibrium of ENR at this $\mathrm{pH}$ range is shown in Figure 5. This combination can destroy the involvement of lone-pair electrons of the 1-position nitrogen in the formation of conjugated $\Pi$ bonds, and significantly affects ENR fluorescence properties.

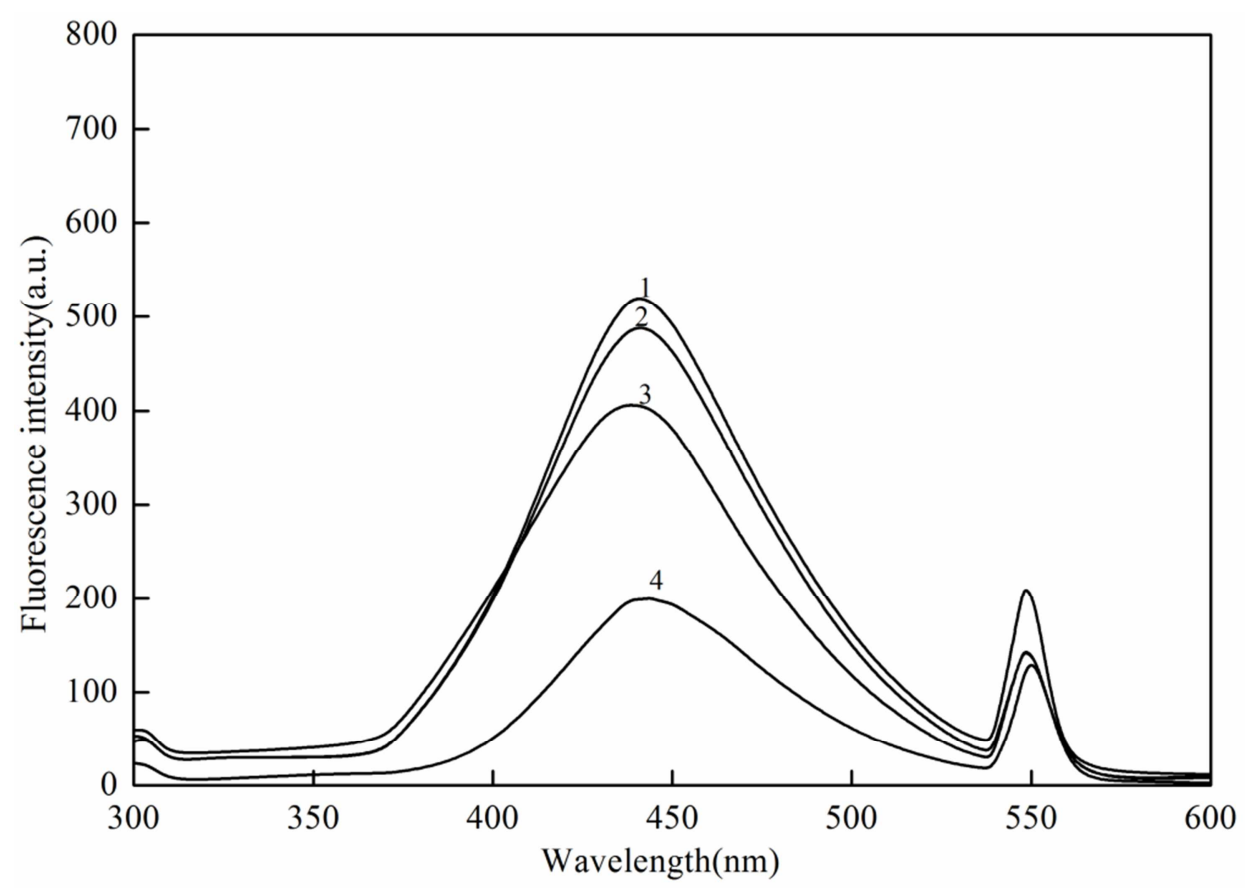

(1) $\mathrm{pH}=2.81$ (2) $\mathrm{pH}=2.45$ (3) $\mathrm{pH}=1.87$ (4) $\mathrm{pH}=1.02$.

Figure 4. Fluorescence spectra of ENR under acidic conditions.<smiles></smiles>

Figure 5. The process of $\mathrm{H}_{3} \mathrm{~L}^{2+}$ dissociation to $\mathrm{H}_{2} \mathrm{~L}^{+}$.

\subsection{Fluorescence Spectra of ENR in Alkaline Conditions}

Figure 6 shows the fluorescence spectra of ENR under alkaline conditions. With increasing $\mathrm{pH}$, the fluorescence intensity at $450 \mathrm{~nm}$ was reduced significantly to the extent that the fluorescence disappears, but the position of fluorescence peak was basically unchanged. This spectral characteristic indicated that the ENR bipolar ion HL gradually lost a proton, and then transformed into $\mathrm{L}^{-}$without fluorescence. As shown in Figure 6, this transition caused a decrease in fluorescence intensity with increasing $\mathrm{pH}$. The proton dissociation equilibrium of ENR at this $\mathrm{pH}$ range is shown in Figure 7. 


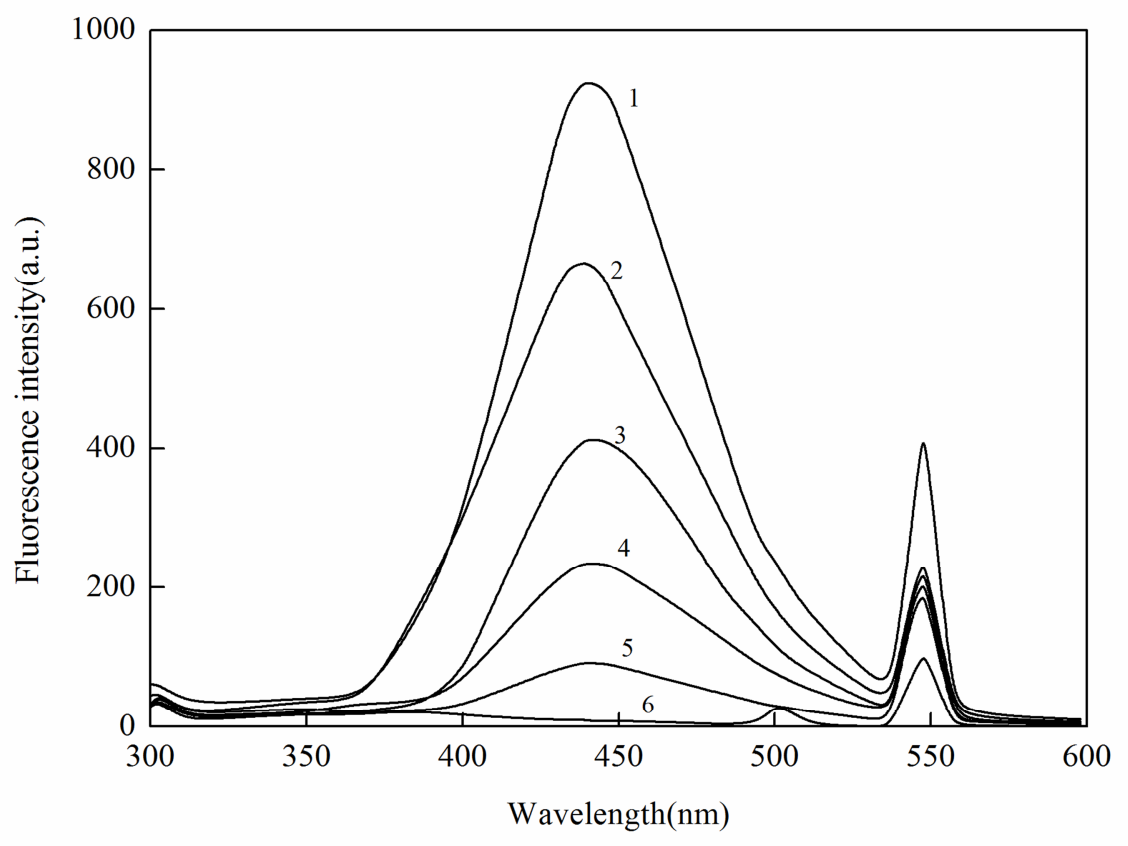

(1) $\mathrm{pH}=9.65$ (2) $\mathrm{pH}=10.04$ (3) $\mathrm{pH}=11.05$ (4) $\mathrm{pH}=11.65$ (5) $\mathrm{pH}=11.95$ (6) $\mathrm{pH}=12.10$

Figure 6. Fluorescence spectra of ENR under alkaline conditions.<smiles>CCN1CCN(c2cc3c(cc2F)c(=O)c(C(=O)[O-])cn3C2CC2)CC1</smiles>

Figure 7. The process of HL dissociated to $L$.

\subsection{Dissociation Constant of ENR}

The dissociation constant $p K_{a}$ of ENR can be calculated based on the following equation [11]:

$$
p K_{a}=p H-\lg \left[\left(F_{H B}-F\right) /\left(F-F_{B}\right)\right]
$$

Where $F_{H B}$ and $F_{B}$ are the fluorescence intensities of ENR completely in the presence of a conjugated acid type or a fully conjugated base type, respectively. $F$ is the fluorescence intensity corresponding to the $\mathrm{pH}$ of solution. According to the above equation, the $p K_{a}$ value can be calculated as long as $F_{H B}$, $F_{B}$ and a set of $p H-F$ data are measured. The calculated data was shown in Table 1 and Table 2. From the data in Table 1, the $p K_{a 1}$ of ENR was $6.80 \pm 0.03$. From the data in Table 2, the $p K_{a 2}$ of ENR was $7.56 \pm 0.10$.

Table 1. The $p K_{a l}$ of ENR at different $p H$ values.

\begin{tabular}{lllll}
\hline $\mathbf{p H}$ & $\mathbf{F}$ & $\mathbf{l g}\left[\left(\mathbf{F}-\mathbf{F}_{\mathbf{B}}\right) /\left(\mathbf{F}_{\mathbf{H B}}-\mathbf{F}\right)\right]$ & $\mathbf{p K}_{\mathbf{a} 1}$ & Average \\
\hline 5.84 & 925 & -0.97 & 6.81 & \\
6.35 & 777 & -0.46 & 6.81 & 6.80 \\
6.82 & 616 & -0.01 & 6.83 & \\
7.14 & 353 & 0.41 & 6.73 & \\
\hline
\end{tabular}

Table 2. The $p K_{a 2}$ of ENR at different $p H$ values.

\begin{tabular}{lllll}
\hline $\mathbf{p H}$ & $\mathbf{F}$ & $\lg \left[\left(\mathbf{F}-\mathbf{F}_{\mathbf{B}}\right) /\left(\mathbf{F}_{\mathbf{H B}}-\mathbf{F}\right)\right]$ & $\mathbf{p K}_{\mathbf{a} 2}$ & Average \\
\hline 10.7 & 312 & 3.19 & 7.51 & \\
11.05 & 298 & 3.45 & 7.60 & 7.56 \\
11.95 & 270 & 4.25 & 7.70 & \\
12.28 & 251 & 4.87 & 7.41 & \\
\hline
\end{tabular}

\subsection{The Fluorescence Quantum Yield of ENR Measurement}

The fluorescence quantum yield is defined as the ratio of the number of photons emitted to the number of photons absorbed $[12,13]$. Fluorescence intensity and the absorbance value of substance and reference substance solutions at the same excitation wavelength were measured, and fluorescence quantum yield of the determined substance was obtained based on the following equation $[14,15]$ :

$$
Y u=Y s \cdot(F u / F s) \cdot(A s / A u)
$$

Where $Y u$ and $Y s$ are the fluorescence quantum yields of substance and reference substance, respectively. $F u$ and $F s$ are the integral fluorescence intensity of substance and reference substance, respectively. $A u$ and $A s$ are the absorbance values of substance and reference substance at excitation 
wavelengths, respectively.

The fluorescence quantum yield of ENR was measured using quinine sulfate as a reference [16-18]. The fluorescence quantum yield of quinine sulfate at the excitation wavelength $313 \mathrm{~nm}$ was 0.55 . ENR solution (adjusted $\mathrm{pH}$ with buffer solution) and quinine sulfate were prepared with absorbance differences of less than 0.05 . The Au and As values were recorded at a specified wavelength, then the fluorescence spectra of ENR and quinine sulfate solutions were scanned at different excitation wavelengths to calculate integrated fluorescence intensity. Finally, the fluorescence quantum yield was calculated according to Formula (2). In this experiment, concentration of ENR, $C_{(E N R)}=1.0 \mu \mathrm{M}$. The quinine sulfate concentration was $4.0 \mu \mathrm{M}$, and the slit width was $3 \mathrm{~nm} / 5 \mathrm{~nm}$. The results were shown in Table 3 .

As shown in Table 3, the fluorescence quantum yield of quinine sulfate was basically the same as at the excitation wavelength of $270-340 \mathrm{~nm}$, and the fluorescence quantum yield of ENR was almost identical as at the excitation wavelength of 250-320 $\mathrm{nm}$. The fluorescence quantum yield of ENR was 0.125 at the maximum excitation wavelength of $274 \mathrm{~nm}$, which indicates that ENR is a relatively strong fluorescent compound.

Table 3. Fluorescence quantum yield of ENR and quinine sulfate.

\begin{tabular}{|c|c|c|c|c|c|c|c|c|}
\hline \multirow{2}{*}{$\lambda / \mathbf{n m}$} & \multicolumn{5}{|c|}{ Quinine sulfate } & \multicolumn{3}{|l|}{ ENR } \\
\hline & $F$ & $A$ & $Y$ & $Y / Y_{313}$ & $\lambda / \mathbf{n m}$ & $F_{E N R}$ & $\boldsymbol{A}$ & $Y$ \\
\hline 270 & 44106 & 0.0099 & 0.49 & 0.89 & 250 & 18250 & 0.015 & 0.134 \\
\hline 275 & 45958 & 0.0107 & 0.47 & 0.86 & 260 & 28364 & 0.021 & 0.123 \\
\hline 280 & 56634 & 0.0131 & 0.48 & 0.86 & 270 & 24571 & 0.024 & 0.124 \\
\hline 290 & 94145 & 0.0196 & 0.58 & 0.96 & 274 & 34753 & 0.024 & 0.125 \\
\hline 300 & 151312 & 0.0303 & 0.55 & 1.00 & 280 & 35490 & 0.020 & 0.132 \\
\hline 313 & 218641 & 0.0437 & 0.55 & 1.00 & 290 & 19732 & 0.011 & 0.131 \\
\hline 320 & 222782 & 0.0443 & 0.55 & 1.01 & 300 & 14348 & 0.006 & 0.128 \\
\hline 330 & 225356 & 0.0440 & 0.56 & 1.02 & 310 & 16607 & 0.005 & 0.141 \\
\hline 340 & 264300 & 0.0524 & 0.55 & 1.01 & 320 & 17476 & 0.005 & 0.126 \\
\hline
\end{tabular}

Integral range of quinine sulfate: $370-590 \mathrm{~nm}$; integral range of ENR: $390-590 \mathrm{~nm}$

\section{Conclusion}

The structure and fluorescence properties of ENR are closely related to the $\mathrm{pH}$ of the solution. When $\mathrm{pH}$ changes, ENR exists in a proton dissociation equilibrium:

$$
H_{3} L^{2+} \Leftrightarrow H_{2} L^{+} \Leftrightarrow H L \Leftrightarrow L^{-}
$$

Under strong acid condition, ENR exists in the $\mathrm{H}_{3} \mathrm{~L}^{2+}$ form of which maximum fluorescence emission wavelength was about $450 \mathrm{~nm}$. At pH 2.45-4.23, ENR mainly exists in the $\mathrm{H}_{2} \mathrm{~L}^{+}$form in which $\mathrm{H}_{3} \mathrm{~L}^{2+}$ loses one proton at the 1-position of nitrogen in the quinoline ring. Fluorescence intensity was strong and steady and maximum emission wavelength was at $450 \mathrm{~nm}$; At $\mathrm{pH}>5.84$, the maximum emission wavelengths were gradually blue shifted to $445 \mathrm{~nm}$ with increase in $\mathrm{pH}$. ENR exists in the form of a bipolar ion $\mathrm{HL}$ in which $\mathrm{H}_{2} \mathrm{~L}^{+}$ loses proton from the carboxyl group. At $\mathrm{pH}>10.04$, HL transforms into the anionic $\mathrm{L}^{-}$form in which $\mathrm{HL}$ loses one proton from the piperazine ring, leading to a decrease in fluorescence intensity. However, the position of maximum emission peak was basically unchanged. The two-step dissociation constant $p K_{a}$ of ENR was calculated, the $p K_{a l}$ was $6.80 \pm 0.03$ and the $p K_{a 2}$ of ENR was $7.56 \pm 0.10$. In buffer solution $\mathrm{pH} 3.00$, with quinine sulfate as reference, the fluorescence quantum yield of ENR at the maximum excitation wavelength of $274 \mathrm{~nm}$ was 0.125 .

\section{Acknowledgements}

The authors gratefully acknowledge the financial support of National Science Foundation of China (Grant no. 21375032).

\section{References}

[1] Wu Y C, Guo S, Dong Q, Song Y Z. Development of an immunochromatographic test strip for rapid simultaneous detection of enrofloxacin and ofloxacin in tissue of chicken muscle and pork. Food Analytical Methods 2016; 9 (10): 2807-2813.

[2] Anirudhan T S, Shainy F, Christa J. Synthesis and characterization of polyacrylic acid- grafted-carboxylic graphene/titanium nanotube composite for the effective removal of enrofloxacin from aqueous solutions: Adsorption and photocatalytic degradation studies. Journal of Hazardous Materials 2017; 324(Pt B): 117-130.

[3] Ftouni H, Sayen S, Boudesocque S, Dechamps-Olivier I, Guillon E, Structural study of the copper (II)-enrofloxacin metallo-antibiotic. Inorganica Chimica Acta 2012; 382(5): 186-190.

[4] Terrado-Campos D, Tayeb-Cherif K, Peris-Vicente J, Carda-Broch S, Esteve-Romero J. Determination of oxolinic acid, danofloxacin, ciprofloxacin, and enrofloxacin in porcine and bovine meat by micellar liquid chromatography with fluorescence detection. Food chemistry 2017; 221:1277-1284.

[5] Yan W, Hu S, Jing C Y. Enrofloxacin sorption on smectite clays: Effects of $\mathrm{pH}$, cations, and humic acid, Journal of Colloid and Interface Science 2012; 372(1): 141-147. 
[6] Zhang W S, Tang B, Liu X, Liu Y Y, Xu K H, Ma J P et al. A highly sensitive acidic $\mathrm{pH}$ fluorescent probe and its application to HepG2 cells. Analyst 2009; 134(2): 367-371.

[7] Wierzchowski J, Sepioł J, Sulikowski D, Kierdaszuk B, Shugar D. Fluorescence emission properties of 8-azaxanthine and its $\mathrm{N}$-alkyl derivatives: Excited-state proton transfer, and potential applications in enzymology. Journal of Photochemistry and Photobiology A: Chemistry 2006; 179(3): 276-282.

[8] Singh R B, Mahanta S, Kar S, Guchhait N. Photo-physical properties of 1-hydroxy-2-naphthaldehyde: A combined fluorescence spectroscopy and quantum chemical calculations. Chemical Physics 2006; 331(2): 373-384.

[9] Fedosov S N, Fedosova N U, Krutler B, Nex E, Petersen T E. Mechanisms of discrimination between cobalamins and their natural analogues during their binding to the specific B12-transporting proteins. Biochemistry 2007; 46(21): 6446-6458.

[10] Park H R, Kim T H, Bark K M, Physicochemical properties of quinolone antibiotics in various environments. European Journal of Medicinal Chemistry 2002; 37(6): 443-460.

[11] Mack J., Vogel P., Jones D., Kaval N., Sutton A., The development of corannulene-based blue emitters. Organic \& Biomolecular Chemistry 2007; 5(15): 2448-2452.

[12] Zhang J, Liu C G., Wei Y J. Fluorescence Quantum Yield and Ionization Constant of Umbelliferone. Chemistry 2011; 74(10): 957-960.
[13] Tian H. J., Tang R. R., Zhao M. M., Synthesis, characterization, and photophysical properties of dinuclear Eu (III) and $\mathrm{Tb}$ (III) complexes based on $\beta$-diketonate with triphenylamine and pyridine moieties. ECS Journal of Solid State Science and Technology 2013; 2(3): R33-R38.

[14] Suzuki K, Kobayashi A, Kaneko S, Takehira K, Yoshihara T, Ishida $\mathrm{H}$ et al. Reevaluation of absolute luminescence quantum yields of standard solutions using a spectrometer with an integrating sphere and a back-thinned CCD detector, Physical Chemistry Chemical Physics 2009; 11(42): 9850-9860.

[15] Liu C G, Xu Y Z, Wei Y J, Qi J, Xu Z H, Ye F et al. Fluorescence spectra and protonation of ofloxacin. Spectroscopy and Spectral Analysis 2005; 25(4): 584-587.

[16] Shelar D P, Rote R V, Patil S R., Jachak M N. Effects of homogeneous media, binary mixtures and microheterogeneous media on the fluorescence and fluorescence probe properties of some benzo [b] $[1,8]$ naphthyridiens with HSA and BSA. Luminescence 2012; 27(5): 398-413.

[17] Drobnik J, Yeargers E. On the use of quinine sulfate as a fluorescence standard. Journal of Molecular Spectroscopy 1996; 19(1-4): 454-455.

[18] Liang X R, Wang G, Jiang Y L, Qu C L, Wang X J, Zhao B. Synthesis and theoretical study on fluorescence property of 4-(2-Hydroxybenzylideneamino)phenyl ethanone schiff base. Spectroscopy and Spectral Analysis 2013; 33(12): 3259-3262. 